\title{
DESCENTRALIZACION Y PARTICIPACION: LOS PARTIDOS Y LOS ACTORES DIRECTAMENTE IMPLICADOS EN LA EXPERIENCIA DE LOS CENTROS COMUNALES ZONALES DE MONTEVIDEO 1990-1992
}

\section{Gerónimo de Sierra (*) / Blanca Charbonnier (**)}

\section{INTRODUCCION (1)}

La tesis central aquí planteada es que las reformas orientadas a la desconcentración de la gestión municipal, así como la propuesta de mayor participación ciudadana y su vinculación con la creación de los Centros Comunales Zonales (CCZ), han sido-en lo fundamental- aceptadas como algo positivo por la mayoría tanto de dirigentes de las comisiones barriales, como de vecinos de Montevideo; sin que ello signifique que hasta el momento se hayan transformado sus hábitos "participativos" en forma profunda y masiva.

También se sostiene, complementariamente, que la aceptación mayoritaria de la propuesta de nueva gestión municipal por parte de la ciudadanía y de los dirigentes de Comisiones barriales, no de la ciudadanía y de los dirigentes de Comisiones barriales, no se debió a la discutible pericia del gobierno en este ámbito de su gestión, ni al inexistente carácter consensual de su aplicación, ni menos aún a un eventual éxito práctico inmediato en la prestación de servicios a los vecinos. Básicamente, dicha aceptación parece explicarse por el hecho de que la propuesta de descentralización y participación -más allá de todas sus ambigüedades y confusiones- interpeló certeramente a la fuerte insatisfacción ciudadana respecto a las formas tradicionales de ejercicio del poder público (2). Y, como factor complementario aunque no menor, al hecho de que los Coordinatorios Zonales (CZ) realizaron una gestión -en lo fundamental- alejada de los "fantasmas" previos sobre un posible accionar de corte ostensiblemente partidista, manipulador o clientelista. Debe reconocerse, que esta orientación de su desempeño tuvo el respaldo explícito del Intendente y del equipo ejecutivo comunal.
Esta interpretación de la causa principal de ese rápido proceso de "aceptación positiva" (ni obvia, ni predecible a priori) que se desprende del conjunto de estas investigaciones, es lo que hace inteligible que la misma se haya generado en un tiempo relativamente breve y a pesar de la serie de obstáculos y dificultades que encontró su puesta en práctica efectiva.

En primer lugar la inexistencia de una tradicción de "politización" (no en su sentido partidista) de los temas y derechos humanos, expresada -entre otros aspectos- por la ausencia de fuertes movimientos sociales reivindicativos de base territorial (3).

En segundo lugar, el fuerte y continuado rechazo a la forma de implementación del proyecto descentralizador por parte de los líderes políticos de la oposición al gobierno municipal.

En tercer lugar -en parte vinculado con lo anterior- el hecho de que la propuesta surgiera de un gobierno de izquierda, con todas las connotaciones y expectativas eventualmente disruptivas que eso implicaba para una parte importante de la población.

Y, en cuarto lugar, el conjunto de dificultades representadas por hechos tales como: los errores y vaivenes del propio gobierno municipal en las propuestas de descentralización y participación (Charbonnier y Pérez Novella, 1992); la lentitud-en cierto modo inevitable- del proceso de desconcentración administrativa; la indefinición relativa del rol de los $\mathrm{CZ}$ y de las normas de funcionamiento de los CZ (Charbonnier, 1992, págs.26 a 29); las rigideces de adaptación del aparato central de la IMM, y en cierta medida los hábitos y estrategias del sindicato de trabajadores municipales.

(*) Profesor de la Facultad de Ciencias Sociales de la Universidad de la Republica Oriental del Uruguay.

(**) Investigadora de CIEDUR.

(1) Para la elaboración de este trabajo se utilizarón resultados de varias investigaciones desarrolladas en el CIEDUR, en el marco del Programa de estudio - dirigido por R.Aguirre y G. de Sierra- sobre los Actores Sociales y Políticos implicados en la experiencia de los Centros Comunales Zonales (CCZ). En especial recoge insumos de las investigaciones realizadas bajo la responsabilidad personal de G. de Sierra:

a) el posicionamiento público de los partidos políticos frente al tema (estudio de prensa); b) el rol y el desempeño de los Coordinadores Zonales (entrevistas en profundidad a siete $\mathrm{CZ}$; c) el funcionamiento y las opiniones de las comisiones vecinales y barriales de diverso tipo que interactúan con los CCZ y con la Intendencia ( encuesta a dirigentes de las comisiones de centro CCZ)

O investigaciones de responsabilidad compartida con R. Aguirre: d) la opinión y las expectativas de los propios vecinos sobre la experiencia en curso (encuesta de opinión pública en los tres CCZ).

(2) Un indicador fuerte de esa actitud ciudadana, puede encontrarse en las respuestas de los montevideanos entrevistados por el CIEDUR (Proyecto Montevideo, Encuesta I y II, 1989 y 1990): "En términos generales ¿en qué grado considera que gente como Ud. tiene posibilidad de incidir en las decisiones que toman....? Los porcentajes de quienes respondieron que "no inciden en nada fueron los siguientes:

• en el gobierno nacional, 74 (1989) y 66 (1990)

- en los partidos políticos, 61(1989) y 62 (1990)

(3) El movimiento social urbano más significativo -a pesar de su relativo decaimiento en los años previos a 1990 - es sin duda FUCVAM. En cuanto a la importante presencia histórica de las Comisiones Vecinales en varios barrios de la ciudad, nos parece indudable que más alla de su significación, ello no habilita a considerarlas un movimiento social. Tanto desde un punto de vista teórico, como en una perspectiva comparativa de los movimientos sociales urbanos en varios países de América Latina y Europa. (Wallerstein et alli., 1990; Machado Da Silva y Torre Ribeiro, 1985; Corragio, 1987) 
En cualquier caso, el conjunto de evidencias empíricas recogidas en las investigaciones realizadas en el CIEDUR, permiten pensar que sin perjucio de las variantes futuras que sufra la experiencia en curso, los aspectos centrales de las transformaciones que se han introducido o impulsado en la forma de encarar la relación entre Intendencia Municipal y los vecinos de Montevideo, tienen "chances" razonables de retener el actual apoyo ciudadano, y por eso mismo, posibilidades de permanecer en el futuro (4). En particular la división de la ciudad en varias zonas, atendidas en forma fuertemente desconcentrada por los CCZ y el departamento de Descentralización; la jerarquización como interlocutores válidos de los diversos tipos de comisiones de base barrial, solas o agrupadas; y alguna forma de delegación de poderes a las Juntas Locales, Comisiones Delegadas y Comisiones Vecinales asesoras, todas ellas eventualmente electas por los "vecinos".

\section{EL CONTEXTO SOCIAL, POLITICO Y CULTURAL, EN QUE LA IMM REALIZA LA PROPUESTA DE PARTICIPACION Y DESCENTRALIZACION}

El fuerte involucramiento de los dirigentes políticos y barriales $-y$ de un importante sector de la opinión pública- en torno a la experiencia de los CCZ, y más ampliamente de las propuestas descentralizadoras y participativas hechas por la nueva administración frenteamplista, tiene su causa fundamental en el contexto general y conyuntural en el que las mismas fueron planteadas y desarrolladas. Ello explica que un tema de muy bajo perfil académico y político hasta pocos meses antes de las elecciones de 1989, haya adquirido considerable centralidad desde que asumió el nuevo gobierno municipal. ¿Cuales han sido las dimensiones básicas de ese contexto?

En primer lugar, el marco general de estancamiento del país, su crisis de horizonte cultural y material, especialmente para los sectores medios y populares; el desencanto considerable de las expectativas que abrió el fin de la dictadura, con su secuela de descreimiento creciente en las formas políticas clásicas y en los propios dirigentes políticos (de Sierra, 1989). Descreimiento que se expresaba - entre otros indicadores- en el descenso de la participación social en general y de la militancia política en particular.

Todo ello acompañado por la inocultable degradación de amplias zonas de la ciudad, tanto en su estructura material, como en su tejido de relaciones sociales tradicionales (Veiga, 1989). Lo cual contribuía a conformar, confusamente, la conciencia de que en los últimos años había emergido una nueva realidad urbana, menos habitable, menos solidaria y mucho mas polarizada que en el pasado (Rodé, 1991). Conciencia que, sin embargo, no lograba expresarse en la aparición de movimientos sociales orientados explícitamente- a la consecución de modelos urbanos alternativos al que se venía imponiendo por los hechos consumados.

En segundo lugar, el impacto provocado en el sistema político y sobre las expectativas ciudadanas, por la victoria del Frente
Amplio (FA) en el departamento de Montevideo. Este impacto tiene una primera base explicativa en el hecho de que era la primera vez que la izquierda ganaba un cargo ejecutivo de esa importancia (de Sierra, 1990), y que ello sucedía en el marco de un descenso significativo del apoyo ciudadano -en la capital y en todo el país- a los partidos tradicionales. Por otra parte, ese impacto se vió reforzado por el contenido del discurso programático del FA, el que dió una prioridad muy alta a la propuesta de democratizar la gestión urbana a través del incentivo de la participación ciudadana y la descentralización territorial de dicha gestión.

En tercer lugar, el hecho de que el gobierno nacional fuera obtenido por el Partido Nacional, con clara mayoría en su seno del herrerismo, el sector nacionalista históricamente mas conservador y más nítidamente comprometido con las políticas de corte neo-liberal en el plano económico y social. Es decir, las más opuestas con el perfil del FA en el discurso programático y en la conciencia ciudadana. Esa circunstancia creaba -per sefuertes expectativas de conflictividad, o por lo menos de rivalidad, en el contenido y los métodos de gobierno de ambas administraciones (5). A ello se agregaba el repliegue obligado del Partido Colorado de uno de sus bastiones históricos de poder y reproducción política, lo que presagiaba un fuerte perfil opositor y polémico por parte de ese partido, tal como efectivamente sucedió hasta el momento (6).

\section{EL DESPLIEGUE PAULATINO DE LA PROPUESTA Y LAS AMBIGUEDADES DEL DISCURSO Y LA PRACTICA OFICIAL SOBRE LA MISMA}

Desde su inicio, el nuevo gobierno se vió atravesado por la discusión sobre el ritmo con que debía de ser aplicado el programa de descentralización y su estructura territorial y político-administrativa. Predominó la tesitura de comenzar de inmediato su instrumentación, creándose rápidamente las 18 circunscripciones, y designado al frente de cada una de ellas un Coordinador Zonal de confianza política del Intendente, aunque en su gran mayoría de bajo perfil político previo.

Mucho más lentamente se fueron conformado las estructuras físicas y administrativas de cada CCZ (y desconcentrando actividades municipales), mientras se elaboraba el decreto -luego retirado y redefinido ante la fuerte oposición política que provocó- de creación de una estructura jurídica específica para el Departamento de Descentralización y los CCZ. Aún más tarde, ya fuertemente influído por los acuerdos de la Comisión Mixta y plural Ejecutivo -Junta Departamental, es que el gobierno pasa a proponer fórmulas concretas de descentralización política (junta Locales y Comisiones Delegadas) y participación formalizada de los vecinos en órganos de consulta taxativa (Comisiones Vecinales, asesoras y consultivas).

Esta decisión de comenzar rápidamente con la llamada "descentralizacion", si bien tuvo la virtud de mostrar la decision

(4) Sin ninguna pretensión de precedir el futuro, parece claro que un eventual gobierno municipal que tratara de desandar lo hecho en este plano, probablemente tendría que ir contra la opinión mayoritaria de la población, y pagar por ello un alto precio político. Así parece indicarlo -entre otros indicadores- el hecho de que $68 \%$ de los vecinos entrevistados en tres CCZ, dicen estar en desacuerdo, o muy en desacuerdo, con "volver a la situación anterior a este gobierno". (ver un análisis detallado de este item en: Aguirre, de Sierra, Iens, Cap.5 de este volumen)

(5) Ver un tratamiento específico y global de estas relaciones intergubernamentales -no sólo sobre el tema CCZ y conexos- en la segunda parte de este volumen; en especial Moreira, Cap.6.

(6) Para un análisis pormemorizado del posicionamiento público de los partidos respecto a los CCZ, los CZ, y más en general respecto a la problemática participacióndescentralización, ver Charbonnier y Pérez Novella, 1992. 
política del gobierno municipal en cuanto a la aplicaciòn efectiva de su programa, le supuso no sólo cometer algunos errores prácticos y hasta jurídicos, sino postergar en el tiempo la inevitable clarificación y desagregación de por lo menos tres dimensiones distintas incluídas en la propuesta genérica inicial: la desconcentración de servicios; la descentralización política y la participación de los vecinos. Todas ellas más o menos confusamente subsumidas por participación por el discurso "oficial" en la ecuación: descentralización $=$ participación $=$ democratización (y a menudo = cogobierno con los vecinos) (7).

El mensaje del ejecutivo comunal -y especialmente del Intendente- se situó desde el inicio en un tono de muy alta jerarquización de este tema como algo estratégico para su proyecto. La lentitud en la clarificación conceptual y en la aplicación de cada una de sus dimensiones, fue sin duda acentuada por las dificultades internas al aparato central de la IMM y al propio FA; así como por la fuerte resistencia de la oposición política, aspecto éste al que nos referimos más adelante.

En especial durante el primer año de gobierno, esta situación compleja depositó sobre los $\mathrm{CCZ}$ y más particularmente sus Coordinadores, una enorme responsabilidad práctica y simbólica. Responsabilidad que durante un período considerable de su gestión, no iba acompañada de los instrumentos jurídicos, materiales y organizativos, correspondientes al desempeño de tan delicada función, con las tensiones y contradicciones consecuentes. A pesar de ello, puede sostenerse que los CZ jugaron, durante el largo período inicial, un papel decisivo en la consolidación dinámica de la propuesta global; especialmente como motivadores y mediadores de la interacción efectiva entre los vecinos y la Intendencia, y como rostro público e inmediato del ejecutivo comunal. Quizás por eso mismo fueron los destinatarios -junto con los propios CCZ- de algunas de las críticas más virulentas partidas desde filas de la oposición; críticas que en lo fundamental no encontraron eco generalizado entre las Comisiones y los vecinos directamente concernidos.

\section{LA OPOSICION NO ESCATIMA SUS CRITICAS}

Dada la novedad de la experiencia "descentralizadora", así como el significado estratégico que el gobierno municipal y el discurso del FA les otorgaron, era previsible que la oposición realizara críticas a la misma. Tanto más, que los cambios ocurridos en el peso electoral de los partidos y su victoria en Montevideo, hicieron que el FA pareciera paulatinamente como elecciones. Por otra parte, las dificultades, zigzagueos y errores de los distintos segmentos de la administración municipal -ya mencionados- favorecieron en parte esa actitud opositora. Sin embargo, un seguimiento minucioso del discurso público de los voceros políticos opositores (Charbonnier y Pérez Novella, 1992), muestra que en general éste adoptó un tono fuertemente agresivo y descalificador, centrándose especialmente en el tema de los CCZ y sus coordinadores. Y ello sin perjuicio de que, a menudo, esos voceros señalaban que ellos y sus partidos estaban globalmente $-\mathrm{y}$ en tesis- de acuerdo con un proceso descentralizador.

\section{FRECUENCIAS DE LAS CRITICAS MAS RECURRENTES A LOS CCZ MENCIONADAS EN LA PRENSA, SEGUN PARTIDO POLITICO DEL OPINANTE. (2.90 al 6. 92)}

\begin{tabular}{lcccc} 
& P.Col. & P.Nal & N.Ecio. & FA \\
\hline Marxismo-Leninismo & 28 & 8 & 1 & -- \\
\hline Partidización & 24 & 11 & 20 & 5 \\
\hline ctés. de Base-reclutamiento & 8 & 13 & 3 & -- \\
\hline Totalitarismo & 5 & 2 & -- & -- \\
\hline Burocratización & 15 & 6 & 3 & -- \\
\hline Ineficiencia & 13 & 2 & 2 & 10 \\
\hline Altos sueldos & 5 & 2 & 5 & --
\end{tabular}

Dejando de lado las críticas de inconstitucionalidad que se expresaron fundamentalmente el primer año (referidas al decreto inicial, luego retirado), resalta el predominio absoluto de críticas que acusan al gobierno, al FA y los CCZ, de utilizar a la descentralización como un pretexto para el manejo político de los vecinos. Pero no un manejo político cualquiera (quizás el "rutinario" y más o menos "tolerable" en toda gestión pública), sino un manejo político inspirado en las utopías revolucionarias, calificado de totalitario, intolerante, marxista- leninista, copiado de las prácticas de la KGB y de los Comités de Defensa de la revolución cubanos. Calificaciones todas ellas que apelan claramente a mecanismos ideológicos de rechazo, que se suponen presentes en amplios sectores de la ciudanía. Por eso mismo es posible afirmar que pretendían descalificar genéricamente el carácter "democrático" del FA.

A estas críticas, se agregan las que asimilan el funcionamiento de los $\mathrm{CCZ}$ a simples Comités de Base frenteamplistas, que operarían como bases de reclutamiento político e ideológico, y como centros de fichaje de los vecinos (10).

(7) Entre muchos otros ejemplos, ver entrevista a Tabaré Vázquez en Cuadernos del Tercer Mundo, Julio, 1991

(8) Como decía uno de los CZ entrevistado a fines de 1990: "en todo este tema de la descentralización, existe un conflicto de quienes tienen cuotas de poder y no las quieren perder. Cada uno en su ámbito, ediles, sindicato, jefes, etc., no están en condiciones de asumir un traspaso e poder hacia la gente, hacia el vecino (...) la posición del CZ es difícil, debe andar con cautela, no tiene respaldo jurídico y aunque esto no pese decisivamente en el trabajo comunitario y el CZ, eso está planteado (...) Es una tarea de gran responsabilidad, pues aparecemos frente a los vecinos y al barrio como la imágen pública concreta de la Intendencia, y a la vez como responsables de todas las fallas del aparato" (Charbonnier, 1992).

(9) Cuadro resumen de los dos años y medio. Ver los datos desagregados por año, en las pags. 26, 51 y 70, de Charbonnier y Pérez Novella, 1992. Aquí se eliminó el item "inconstitucionalidad" por ser una crítica referida al decreto inicial, luego retirado. También se agruparon "reclutamiento" y "proselitismo".

(10) Algunos ejemplos, entre otros muchos, de ese tipo de cuestionamientos son los siguientes:

-El Semanario Jaque del 24.4.90, identifica a los CCZ con la utopía revolucionaria, La Habana de Fidel, el Chile de Salvador Allende y un proyecto Leninista.

-Silmaldone el 8.8.90, dice que el modelo de los CCZ son los CDR cubanos y que no le extrañaría que Tabaré Vazquez se dejará la barba y se declarara marxista-leninista.

-Armonía Supparo el 3.11.90 afirma que la gestión municipal de Tabaré Vázquez se está perfilando como un centralismo burocrático de tono monárquico, al estilo soviético.

-Silmaldone dice el 10.2.91, que hay pruebas evidentes de que los $\mathrm{CCZ}$ se han politizado y convertido en comités de base freenteamplistas y el 8.5 .91 que usan métodos totalitarios, hacen demagogia y facilitan la infiltración comunista.

-García Pintos dice el 14.6.91 que la descentralización está disfrazada y que el objetivo real es la politización extrema.

-El presidente Lacalle afirma el 2.7.91 que toda la gestión está politizada y es totalitaria.

-Hierro López sostiene el 19.9.91 que los CCZ, son entes totalmente burocratizados y politizados. 
Si bien es del Partido Colorado -globalmente consideradode donde surgen las críticas más virulentas y contínuas, en grados diversos ellas están presentas también en el Partido Nacional y el Nuevo Espacio (sobre todo el PGP), en ese orden.

Respecto a las críticas de politización general de la experiencia, de burocratización, de ineficiencia, de excesos de gastos, etc., ellas son comunes a los tres agrupamientos opositores casi sin distinción (Charbonnier y Pérez Novella, 1992)

Es evidente -más allá de que una parte de estas críticas puedan atribuirse a fines de retórica opositora con miras a las futuras elecciones- ellas representan también un intento de influir -aquí y ahora- en los comportamientos y las actitudes de las bases electorales de esos partidos respecto a los $\mathrm{CCZ}$ y el conjunto del proceso de descentralización y participación. Bases que en un principio representan más de la mitad de la población del departamento. Como lo analizamos en el inciso siguiente, los hechos mostraron que los "vecinos" y los dirigentes de las Comisiones barriales de diverso tipo, están muy lejos de compartir esas opiniones de sus dirigentes políticos.

Porque escapa al núcleo central de nuestro tema, no analizamos aquí las críticas más generales al Intendente y al conjunto de su gestión al frente de la IMM. Cabe sí recordar, que un sinnúmero de encuestas han mostrado en forma inconstrastable el bajo impacto de las mismas en la opinión pública.(11)

\section{LA EVIDENCIA EMPIRICA DE LA ACEPTACION POSITIVA DE LA EXPERIENCIA DE "DESCENTRALIZACION - PARTICIPACION" Y LOS CCZ}

En los informes respectivos sobre encuestas a vecinos y a los dirigentes de las Comisiones barriales (Pérez Novella y Charbonnier, 1992), puede verse el análisis desagregado de una serie de indicadores que muestran -en ambos universosla ya mencionada actitud predominante de aceptación básica de varios de los rasgos centrales de la experiencia en curso. Aquí sólo presentaremos algunos datos agregados y altamente expresivos de esa tendencia, con la intención de apoyar empíricamente la afirmación de que ha existido un evidente desfasaje entre, por un lado, el discurso predominante en buena parte de los dirigentes políticos opositores, y por otro, la mayoría de la población -incluyendo sus propios simpatizantes- así como los dirigentes de Comisiones barriales.

Si bien hay variantes según el tipo de comisión y en las distintas categorías de vecinos (edad, sexo, nivel educativo, preferencia política, nivel socio-económico, $\mathrm{CCZ}$ en el que viven), esas diferencias no llegan a modificar el sentido general de nuestro análisis. En lo que hace a las preferencias políticas de los vecinos -variable que resultó casi siempre la mas discriminante respecto a este tema- si bien entre los simpatizantes del Partido Colorado es donde se encuentran siempre los me- nores guarismos de apoyo (u oposición, según como se formularon las preguntas), igual se constata un decalage dignificativo con el total de "colorados" existentes en el momento de la encuesta.

Comenzando por los temas más "generales", encontramos que el $85 \%$ de los vecinos entienden positivo participar en las actividades del CCZ (aunque luego no lo hagan efectivamente por diversos motivos), y que al $82 \%$ le parece importante tratar de participar de alguna manera en la solución de los problemas urbanos en los que tiene ingerencia la gestión de la IMM. Son cifras muy significativas en tanto muestran una clara apertura -o al menos el no cerramiento previo- a la interpelación participativa emitida desde el gobierno comunal. Y ello, a pesar de las acervas críticas emitidas por dirigentes que presentan -sumados- a la mayoría de la población de Montevideo; y al hecho de que luego sólo el $40 \%$ de los vecinos y el $33 \%$ de los dirigentes de Comisiones, piensen que efectivamente participan en algún grado en la toma de decisiones concretas de la IMM.

Por otro lado, el $72 \%$ de los dirigentes de Comisiones piensan que la apertura del CCZ y la presencia del CZ, fué importante para el funcionamiento y la eficacia de su labor. Mientras que el $53 \%$ de las Comisiones ya realizan sus gestiones y demandas directamente ante el CCZ, contra sólo un $13 \%$ que continúan remitiéndose a las oficinas centrales de la IMM. A su vez, el $52 \%$ de los vecinos hace balance general positivo -en algún grado- de lo actuado por su $\mathrm{CCZ}$, contra sólo un $9 \%$ que piensan que fue negativo. a los efectos del análisis específico del contraste entre las severas críticas de los dirigentes y sus "bases" -tema que ahora nos ocupa- es también significativo que el $39 \%$ no tenga ninguna opinión a ese respecto y que el $43 \%$ no tenga todavía un criterio formado sobre aquello que no deberían hacer los CCZ.

Ya referido más específicamente a la actitud participativa de las Comisiones barriales ( $45 \%$ de las cuales nacieron después de marzo de 1990, fecha en que el FA asume el gobierno) en la nueva estructura en desarrollo -a pesar de todas las debilidades, incomunicaciones y trabas de esta última- cabe señalar que el $65 \%$ de aquellas querrían poder participar en plenarios para decidir sobre los múltiples temas conflictivos que debe procesar el $\mathrm{CCZ}$ y eI MM. Y que el $62 \%$ de las Comisiones querrían poder elegir al Coordinador de su CCZ, en caso de que ello les fuera permitido legalmente (12).

Finalmente, y para no extender aquí esta breve recapitulación, nos limitaremos a complementar lo mencionado en la llamada $\mathrm{N}^{\circ} 4$. Si el $68 \%$ del total de vecinos de los tres CCZ estudiados declaran su desacuerdo con "volver a la situación anterior a este gobierno", es quizás aún mas elocuentes que ese total se componga de un $49 \%$ de colorados, un $60 \%$ de blancos, un $78 \%$ de nuevoespacistas, y como era esperable, un $87 \%$ de frenteamplista (13). En cuanto a la distribución por grupos etarios de ese desacuerdo, se constata que mientras la población más envejecida (56 años y más) rechaza la vuelta atrás en un $51 \%$, todos los otros grupos etarios lo hacen entre un 72 y $74 \%$.

(11) Como se sabe, el raiting general de aceptación de Intendente tendió a estabilizarse en valores de mas del 50\%. En nuestra encuesta a los vecinos de tres CCZ, su gestión global obtiene una aceptación de $57 \%$.

(12) En el caso de los vecinos -entrevistados cuando ya lo que estaba en juego efectivamente era la eventual elección de las Juntas Locales y Comisiones Delegadas- éstos se manifestaron abrumadoramente por participar en la elección de sus integrantes (62\%) y contra la designación directa por parte del Intendente (24\%)

(13) Cada vez que en este trabajo nos referimos a la variable "simpatía política", debe tenerse en cuenta que hemos utilizado como indicador el voto que el entrevistado declaró haber emitido en noviembre de 1989. 


\section{EL IMPACTO SOBRE LOS ACTORES IMPLICADOS Y SUS RELACIONES MUTUAS}

Es indudable que más allá de cual sea el destino definitivo de la experiencia en curso, desde ya ella ha impactado fuertemente a todos los actores involucrados, presionándolos a revisar sus formas tradicionales de relacionarse entre sí para el cumplimiento de sus roles respectivos. En realidad son otros propios roles los que se van modificando paulatinamente, no sin tensiones y disfuncionalidades varias.

a) A comenzar por el propio aparato central de la IMM (a nivel político-ejecutivo y administrativo), el que estaba moldeado por reglamentos y prácticas fuertemente centralizadas de larga data. No era solamente la novedad o la inercia burocrática, lo que generó y aún genera las dificultades de relación entre las demandas locales "elevadas" por los CZ, y su tratamiento eficaz en tiempo y forma por parte del aparato central. Mas allá de la defensa de espacios de poder decisional de parte de los distintos escalones de este último, lo que se produjo fue una dificultad efectiva para comprender los desafíos de una gestión unificada pero perceptiva a las demandas priorizadas localmente por los vecinos, y legitimadas por el CCZ. Contrastando con el discurso emitido desde la cúpula, muchos escalones decisorios centrales jefes de Departamentos, de Servicios, Capataces, etc,- seguían funcionando con la "vieja cabeza".

La reforma estructural de los Departamentos, la paulatina desconcentración de servicios, la continuada demanda de los $\mathrm{CZ}$ (a su vez presionados por los vecinos y Comisiones) y la paulatina clasificación del diseño descentralizador por parte del Ejecutivo comunal, va generando condiciones de gestión que parecen aproximarse más al espíritu pregonado de la reforma. En cualquier caso, es en este plano donde se jugará a largo plazo buena parte del éxito de la interpretación descentralizadora y participativa, poniendo a prueba su credibilidad política y sus chances de durabilidad sustantiva y no sólo formal-jurídica.

b) Las Comisiones Barriales de diverso tipo fueron influídas muy directamente por la paulatina implantación de la nueva estructura de relacionamiento vecinos- Intendencia. En primer lugar por el propio "clima" más directo y comunicativo creado por el estilo del Intendente, la comparecencia periódica de todo el gabinete a los barrios, y sobre todo por la convocatoria a participar, proponer, controlar, e incluso a "cogobernar", emanada desde el Ejecutivo comunal. A ello se agregó la presencia directa y permanente de los Coordinadores en el CCZ, así como la paulatina desconcentración de servicios.

Habituadas a una acción muy circunscripta a reclamos puntuales y "micro locales", ante oficinas y funcionarios de la IMM o los ediles, se vieron confrontadas simultáneamente a dos nuevas realidades. Por un lado la posibilidad $-\mathrm{y}$ poco a poco la necesidad- de dirigirse al eslabón local de la Intendencia representado por el CCZ, una unidad con más conocimiento directo de las necesidades, pero al mismo tiempo con recursos administrativos y decisorios aún limitados, y por lo tanto, lentos. Y, por otro lado, a la paulatina necesidad de confrontar sus demandas a las de las otras comisiones y vecinos de la zona -a menudo tan legítimas como las propias, pero eventualmente contradictoriasy de esa manera se vieron enfrentadas al aprendizaje de la globa- lización, del control de otros vecinos, y de la evaluación "pública" de la factibilidad y racionalidad de sus reclamos.

El conjunto de todas estas nuevas contextualidades -sumadas a la creación eventual de Comisiones Asesoras electas localmente- va enfrentando a las Comisiones barriales a la doble tarea de reafirmar su legitimidad y eficiencia por un lado, y por el otro a buscar formas de articulación mutua que les permita generar mecanismos de representación de intereses comunes a todas ellas. Un saldo de este nuevo sistema operacional es poner en jaque las tendencias clásicas a la heteronomía y al clientelismo político manifiesto, y potencialmente generar condiciones para la aparición de movimientos sociales basados en el "lenguage de los derechos urbanos" y capaces de vehicular -en el espacio de lo público- las dimensiones de la vida cotidiana y la diversidad e identidades propias a la vida local, ampliando de esa manera las dimensiones del concepto y la práctica de la ciudadanía (Silva, 1990; Evers, 1984).

Ligado a esta problemática, queda planteada la interrogante de como se verá influida la relación tradicional entre las Comisiones y el conjunto de los vecinos, por la eventual concreción de Juntas Locales (o Comisiones Delegadas) y las Comisiones Consultivas, todas ellas electivas según el proyecto del gobierno municipal. A pesar de que mayoritariamente las Comisiones actuales son integradas por un número reducido de miembros, en general auto-cooptados, ellas son consideradas mayoritariamente como "representativas" por parte de los vecinos. Sin embargo -de concretarse las elecciones locales en sus dos niveles- surgirá una doble fuente de legitimidad y esa representatividad sui géneris puede verse cuestionada por un nuevo actor, elegido justamente para representar a los vecinos y las Comisiones en las instancias decisorias y consultivas del nuevo poder descentralizado (Villasante, J. R., 1992). Esta problemática específica es la que hace emerger quizás con más nitidez, las zonas imprecisas del discurso "oficial" respecto a las relaciones entre descentralización, participación y "gobierno por los vecinos".

c) En cuanto a las fuerzas políticas, puede decirse que todas ellas -aunque en forma diversa- fueron descolocadas y desbordadas por el proceso de puesta en marcha de la "descentralización" y la creación de los CCZ. La dinámica política y social objetiva generada, pareció haber superado sus expectativas, inclusive de las fuerzas integrantes del FA. Esto es válido para las dirigencias políticas centrales, pero también para sus adherentes, votantes y hasta militantes.

En el caso de los partidos tradicionales, mas allá de la creación por ambos de organismos políticos destinados al seguimiento local de la experiencia de los CCZ (cuya actividad y efectividad es dificil de evaluar), parece sobre todo haberse producido un gran temor por el efecto, real o supuesto, que la nueva experiencia -dirigida por la izquierda- pudiera ocasionar sobre sus mecanismos tradicionales de influencia y cooptación política a nivel barrial. El análisis de sus intervenciones públicas (organismos, parlamentarios, ediles), los muestran jerarquizando más bien la filosofía global del proyecto y la necesidad de su control político en la cúpula, que analizando con detalle el funcionamiento de cada CCZ, sus limitaciones, errores efectivos, el grado de eficiencia, etc. Es cierto que la descentralización de los nucleos de interacción entre los vecinos y la Intendencia, supone

(14) Vecinales y de Fomento, de Complejos Habitacionales o similares, y las que funcionan directamente vinculadas a instituciones diversas como clubes, poloclínicas, parroquias, etc. (15) Ante la pregunta, ¿Considera Ud. que las organizaciones barriales son representativas de los vecinos?, las respuestas fueron: Que sí un 51\%; Más o menos un 19\%; Que no un $13 \%$, y dijeron no tener posición un $17 \%$. (Ver detalle en Cap. 5 de este volúmen) 
un numeroso y complejo aparato de seguimiento y evaluación, para lo cual no estaban preparados.

Tampoco el FA -a pesar de la apariencia o las expectativas apriori- pudo sacar las conclusiones prácticas que imponía la trascendencia de sus propuestas, ahora aplicadas desde el gobierno. Más allá de que todos sus sectores apoyaran en general la consigna descentralización -participación, en la práctica ninguna de sus fuerzas políticas- ni siquiera las más abanderadas en el tema -llegaron a destinar al trabajo barrial en los CCZ la cantidad y calidad de fuerzas que podría esperarse de su discurso. En parte este hecho puede atribuirse al momento de particular desafección militante, pero en realidad eso no explica el que tampoco las direcciones políticas (ya sea de cada partido o colectivas del FA) dedicaran un tiempo calificado al seguimiento y definición de directivas precisas hacia los frenteamplisimas que, ya sea en la base o en cargos de responsabilidad, estuvieron directamente implicados en la experiencia.

Más bien, todo parece indicar que aquello que fué puesto en cuestión son las formas tradicionales en que los partidos en cuanto tales -todos ellos- piensan y operan sobre lo político; en particular su relación con los acuciantes problemas de la vida cotidiana, con la heterogeneidad de las experiencias locales, y ejes emergentes de la formación de clivajes e indentidades en la sociedad montevideana. Esto parece especialmente cierto respecto a la realidad cotidiana vivida por amplios sectores medios en creciente orfandad de proyecto cultural y de vida, sobre todo respecto a los sectores populares, en rápido proceso de desarticulación de las viejas identidades.

Analizado por sus efectos, parecería como si gran parte de la elite política hubiera perdido la capacidad de escucha de las nuevas, heterogéneas y hasta dramáticas demandas que surgen del nuevo tejido social y cultural emergente en el sector popular de la sociedad civil montevideana; demandas cada vez más lejos de ser atendidas por un Estado crecientemente prescindente y por un sistema de partidos que le cuesta superar las formas de mediación entre política y sociedad tradicionales en el país.

Para concluir, permítasenos citar in extenso estas pertinentes reflexiones de José Nun (1991: 23-25): "Por cierto, no se trata de fetichizar la participación, ni de convertirla tampoco en un nuevo deus ex machina. La participación es una de esa categorías que se conocen como "esencialmente controvertidas" y no un talismán. Las identidades y las autonomías que puede promover en el lugar de trabajo o en el barrio, o en cualquier otro espacio, ni son absolutas ni se constituyen de una vez para siempre: se redefinen y se negocian y, por lo tanto, es inherente a ellas el conflicto. Se trata, en cambio, de superar los supuestos restrictivos sobre los que ha descansado la concepción ortodoxa del liberalismo democrático, desde Shumpeter en adelante. Por eso es importante poner énfasis en que el rechazo del principio de representación como criterio exclusivo de funcionamiento, o del atomismo individualista como fundamento pre-teórico, o de la esfera gubernamental como único espacio de la política, no significa de ninguna manera abandonar las garantías constitucionales, o el principio de separación de poderes, o los derechos de ciudadanía, o -inclusive- la distinción misma entre los espacios del Estado y la sociedad civil (...). En situaciones transicionales como las que enfrentan la mayoría de los países a que me refiero (los que viven las transiciones post dictaduras), es casi imposible una consolidación a largo plazo de una democracia representativa digna de su nombre si no se expande la participación, esto es, si no se impulsa una amplia deliberación entre iguales acerca del mejor uso de los recursos de la sociedad; del tipo de modernización que se prefiere; de cuales son las necesidades prioritarias y del modo de satisfacerlas; de como deben definirse lo público y lo privado; del "mix" más conveniente entre las pautas de centralización y de descentralización en la toma de decisiones; etc."

Porque compartimos en todos sus términos estas consideraciones, pensamos que -más allá de su corta duración y de sus claroscuros y ambigüedades- es posible que el éxito subjetivo alcanzado por la experiencia en curso, sea un indicador de las reservas democráticas de amplios sectores de la sociedad, y por lo tanto una expresión embrionaria -aunque sea simbólica- de la posible constitución de un nuevo proyecto de ciudad y de país. Proyecto en el cual política y sociedad no sólo vuelvan a articularse, sino que logre implicar a todos los habitantes, en particular a los grandes bolsones de ciudadanos crecientemente segregados y cada vez más incrédulos en sus dirigentes.

\section{REFERENCIAS BIBLIOGRAFICAS}

- Aguirre, R., de Sierra, G.e Iens, I., "Informe de una encuesta a vecinos sobre Descentralización, Participación y CCZ". CIEDUR, Serie Investigación, N ${ }^{\circ} 99$, Montevideo (y Cap.5 de este volumen).

- Charbonnier, B., 1992- "Análisis de entrevistas en profundidad a siete Coordinadores Zonales". CIEDUR, Serie Investigaciones, $\mathrm{N}^{\circ} 97$, Montevideo.

- Charbonier, B. y Pérez Novella, 1992.- "Posicionamiento público de los actores políticos, sobre los CCZ, participación y descentralización municipal; 1990-1992". CIEDUR, Serie Investigaciones, $\mathrm{N}^{\circ} 100$, Montevideo.

- Coraggio, J.L. (Com.), 1997- La investigación urbana en América Latina. Las ideas y su contexto. Ed. Ciudad de Quito, Quito.

- De Sierra, G., 1989 - "Los problemas de la transición. ¿Hacia dónde vamos?", CIEDUR, Uruguay Hoy, 4a. Serie, Montevideo.

- De Sierra, G., 1990- "Por primera vez en la historia, gobierno de izquierda en Montevideo". Ciudad Alternativa, Año 2, $\mathrm{N}^{\circ}$ 3, Quito.

- Evers, T., 1984 - "Identidaes: a face oculta dos novas movimientos sociales". Novos Estudos, №4, vol. 2, abril, Sao Paulo.

- Machado da Silva, L.A. y Torres Ribeiro, A.C., 1985 "Paradigma e movimiento social: por onde andam nossas idéais?". In: Ciencias Sociais Hoje, 1985, Cortez -ANPOCS, Sao Paulo.

- Nun, J., 1991- "La democracia y la modernización, treinta años después". Ponencia presentada en el XV Congreso Mundial de la Asociación Internacional de Ciencia Política, Buenos Aires. Julio 21-26.

- Pérez Novella, Ma. J. y Charbonnier, B., 1992 - 'Encuesta a dirigentes de Organizaciónes Barriales sobre la experiencia de descentralización-participación y los CCZ". CIEDUR, Serie Investigaciones. $\mathrm{N}^{\circ} 96$, Montevideo. 
- Rodé, P., 1991 -"Montevideo, involución o esperanza". Nueva Sociedad, $\mathrm{N}^{\circ} 114$, julio-agosto, Caracas.

- Silva, A.A., 1990 -"A luta pelos direitos urbanos: novas representacoes de cidade e ciudadania". Espaco e Debates, NERU, año X, N³0, Soa Paulo.

- Veiga, D., 1989 - "Segregación económica y crisis urbana en Montevideo". In: Las ciudades en conflicto. CIESU-EBO, Montevideo.
- Villasante, T.R., 1992 -Las tensiones entre descentralización y participación; la experiencia española. Mimeo, Montevideo.

- Wallerstein et alli., 1990 -El juicio al sujeto. Un análisis global de los movimientos sociales. FLACSO- Miguel Angel Porrúa, México.

\title{
RESUMEN
}

En el presente artículo los autores analizan la experiencia de implementación de mecanismos de descentralización administrativa con ampliación de la participación ciudadana que está siendo puesto en práctica en la actualidad en Montevideo por el gobierno municipal del Frente Amplio, y el importante apoyo que ha recibido por parte de la ciudadanía.

\begin{abstract}
In the present artycle, the authors analyze the experience of implementation of administrative decentralization mechanisms with implication of the citizen participation, which is being imposed nowadays in Montevideo by the Municipal Goverment of Frente Amplio, and the important support wich has received by the citizenship.
\end{abstract}

